\title{
Surgical treatment of infective endocarditis in intravenous drug abusers
}

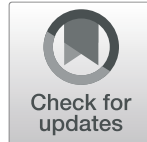

\author{
Alina Zubarevich ${ }^{1 *}$ (D), Marcin Szczechowicz', Anja Osswald', Jerry Easo ${ }^{1}$, Arian Arjomandi Rad², Robert Vardanyan², \\ Bastian Schmack', Arjang Ruhparwar', Konstantin Zhigalov and Alexander Weymann
}

\begin{abstract}
Background: Despite current progress in antibiotic therapy and medical management, infective endocarditis remains a serious condition presenting with high mortality rates. It also is a life-threatening complication in patients with a history of chronic intravenous drug abuse. In this study, we analyzed our institutional experience on the surgical therapy of infective endocarditis in patients with active intravenous drug abuse. The aim of the study is to identify the predictive factors of mortality and morbidity in this subgroup of patients.

Methods: Between 2007 and 2020, a total of 24 patients (7 female, mean age $38.5 \pm 8.7$ ) presenting with active intravenous drug abuse underwent a surgical treatment for the infective endocarditis at out center. The primary endpoint was survival at 30th day after the surgery. The secondary composite endpoint included freedom from death, recurrent endocarditis, re-do surgery, and postoperative stroke during the follow-up period. Mean follow-up was $4.2 \pm 4.3$ years.
\end{abstract}

Results: Staphylococcus species was the most common pathogen detected in the preoperative blood cultures. Infection caused by Enterococcus species as well as liver function impairment were identified as mortality predictor factors. Logistic EuroSCORE and EusOSCORE-II were also predictive factors for mortality in univariate analysis. Survival at 1 and 3 years was 78 and $72 \%$ respectively. Thirty-day survival was 88\%. 30-day freedom from combined endpoint was $83 \%$ and after 1 and 3 years, 69 and 58\% of the patients respectively were free from combined endpoint. Five patients (20.8\%) were readmitted with recurrent infective endocarditis.

Conclusion: In patients presenting with active intravenous drug abuse, treatment of infective endocarditis should be performed as aggressively as possible and should be followed by antibiotic therapy to avoid high mortality rates and recurrent endocarditis. Early intervention is advisable in patients with an infective endocarditis and enterococcus species in the preoperative blood cultures, liver function deterioration as well as cardiac function impairment. Attention should be also payed to addiction treatment, due to the elevated relapse rate in patients who actively inject drugs. However, larger prospective studies are necessary to support our results. As septic shock is the most frequent cause of death, new treatment options, e.g. blood purification should be evaluated.

Keywords: Infective endocarditis, Intravenous drug abuse, High risk valve procedures

\footnotetext{
*Correspondence: alina.zubarevich@gmail.com

'Department of Thoracic and Cardiovascular Surgery, West German Heart and Vascular Center, University of Duisburg-Essen, Essen, Germany

Full list of author information is available at the end of the article
} 


\section{Background}

Despite numerous anti-drug initiatives, the global rate of intravenous drug abuse has been sharply rising, with an estimated 15.6 million worldwide users in 2015 [1].

Intravenous drug abusers are at an increased risk of infective endocarditis (IE), due to the bacteremia caused by unsterile injections. Their population is mostly constituted by relatively healthy young adults with no underlying cardiac diseases. Nevertheless, despite the patients' young age, IE presents itself as a challenging condition with high mortality rates. Overall, even in the era of modern medicine with targeted antibiotic therapy IE in patients with active intravenous drug use (IVDU) presents with 1-year mortality rates of up to $40 \%$ [2]. Approximately 60 to $70 \%$ of cases require cardiac surgery, which carries an extremely high risk due to the often critical risk posed by sepsis or congestive heart failure. Furthermore, the rate of recurrent IE remains high. Additionally, most cases of IE in IVDU are due to multiple antibiotic resistant and highly virulent organisms, thus having a negative influence on the effectiveness of antibiotics and leading to IE recurrences [2, 3]. The surgeons' eagerness to perform surgeries on patients with active IVDU is cooled down due to the noncompliance of the patients and high rates of relapsing on the drug use. Unfortunately, when being provided surgical and medical therapy to prevent recurrence of IE, not all of IVDU patients receive appropriate addiction treatment and psychiatric counselling, especially those patients who are actively injecting.

In this study we sought to review our institutional experience with the surgical treatment of patients with IE and active IVDU, to contribute to the limited number of studies available on this issue to identify the predictive factors of mortality and to review our strategies of care in this particular patient cohort.

\section{Methods}

\section{Study design and patient cohort}

Between 2007 and 2020, a total of 24 patients (7 female, mean age $38.5 \pm 8.7$ ) presenting with intravenous drug use (IVUD) underwent surgical treatment for infective endocarditis (IE) at our center. The patients enrolled received a diagnosis of IE according to the modified Duke criteria [4] and were current intravenous drug users until the hospital admission. Data collected prospectively as a part of the institutional database included detailed information on patients' demographics, baseline clinical characteristics, their laboratory tests, preoperative medical history, echocardiographic and hemodynamic parameters, as well as intraoperative variables and postoperative outcomes. The study was approved by the local ethic committee. All patients signed the informed consent on follow-up. Follow-up was performed via telephone interview with the patients' GPs and/or the patients. The data on mortality was provided by the local city hall bureau of vital statistics if the information was unobtainable from the medical records.

The primary endpoint in this study was survival at 30th day after the surgery. The secondary composite endpoint included freedom from death, recurrent endocarditis, re-do surgery, and postoperative stroke during the follow-up period. The aim of the study was to evaluate our institutional experience of IE in IVDUs and to identify factors influencing morbidity and mortality in this cohort.

\section{Diagnostic criteria and indications for surgical treatment}

The diagnosis of IE was made using a combination of laboratory tests, clinical findings and the information acquired from transesophageal echocardiography, according to the modified Duke criteria. During preoperative work-up, all the patients underwent abdominal ultrasound and a full body CT-scan to obtain information about the septic foci. Absolute indications for the urgent of emergent procedure were: prosthetic valve endocarditis, progressive heart failure, unmanageable infection despite optimal antibiotics, septic embolism and acute renal failure. Each patient in our study matched at least one of those criteria.

\section{Surgical technique}

All procedures were performed on cardiopulmonary bypass with mild hypothermia. Myocardial protection was achieved with antegrade cold crystalloid cardioplegic solution. Mitral- and tricuspid valve procedures were performed with occlusion of both caval veins. Mitraland aortic valve procedures were performed on the arrested heart and isolated tricuspid valve procedures were performed either in cardiac arrest or on beating heart according to surgeons' preferences.

The treatment concept of the IE in our institution implies radical debridement of the infected tissue followed by irrigation with antibiotic solution. All visibly infected and necrotic structures were resected and all the abscesses were evacuated and if needed were filled with gentamicin-glue and covered with bovine pericardial patch (St. Jude Medical, Saint Paul, Minnesota, USA). Valve repair was preferred over valve replacement if enough of the valve anatomy was intact after the debridement. Defects were closed with pericardial patches or if possible, by direct stitches. The use of the foreign materials was avoided when possible.

In cases of valve replacement, the type of chosen prosthesis was generally determined by each surgeons' preference together with patients' wish. Prosthesis, sutures and patch material were soaked in gentamicin solution before use. 


\section{Follow-up}

Follow-up was performed either by telephone interview with the patients' general practitioners who also performed the postoperative echocardiography, or by telephone contact with patients and/or family members. The data on mortality was provided by the local city hall' bureau of vital statistics if the information was unobtainable from the medical records.

\section{Postoperative care}

An important goal of postoperative therapy was controlling the local and systemic inflammatory process. In general, broad spectrum antibiotic therapy was initially started which was then directed against the infecting microorganisms. If there was a rapid decline of the infective signs, we deescalated the therapy. If no microorganisms were detected, a broad calculated triple therapy with imipenem and vancomycin was applied. In every case, intravenous antibiotic therapy was continued for 6 weeks postoperatively.

Transesophageal and transthoracic echocardiography were performed regularly over the course of treatment to exclude signs of the recurrent vegetations or paravalvular leakage. The latter, in combination with persisting signs of infection would constitute an indication for reoperation due to the strong suspicion of a recurrent IE.

\section{Statistical analysis}

Surgical treatment technique was not randomized, it was rather determined by the best medical judgment based on each individual case. Data was collected from chart reviews. Statistical analysis was performed using IBM SPSS version 26 (IBM Corp., Chicago, Ill., USA) and R software v.3.4.3 (R Foundation for Statistical Computing, Vienna, Austria). The data was checked for normality using the Shapiro-Wilk test. If data was not normally distributed, continuous variables are expressed as the medians (interquartile range, IQR). Categorical variables are expressed as frequencies and percentages. We used the Kaplan-Meier method to analyze survival. The significance of survival differences between the groups was assessed with Log-Rank and Breslow tests. A value of $p<0.05$ was considered to be statistically significant.

\section{Definitions}

Septic shock - a subset of sepsis in which particularly profound circulatory, cellular, and metabolic abnormalities [5]. Acute kidney failure on dialysis - a rapid fall in the rate of glomerular filtration, which manifests clinically as an abrupt and sustained increase in the serum levels of urea and creatinine with an associated disruption of salt and water homeostasis treated on dialysis [6].

\section{Results}

The mean age of the cohort was $38.5 \pm 8.7$ years with $29.2 \%$ of the patients being female (Table 1 ). All patients in the study were active intravenous drug users at the moment of admission. Previously, one of the patients underwent a tricuspid valve replacement procedure due to tricuspid valve endocarditis. Further medical history included Hepatitis C (79.2\%), kidney injury (29.2\%), liver cirrhosis (16.7\%) and HIV (12.5\%). Blood cultures were positive in $70.8 \%$ of the patients with Staphylococcus aureus identified in $82.4 \%$ of them. Infection focus was unknown in $70.8 \%$ of the patients. Prior to the admission, $62.5 \%$ of the cohort suffered septic embolism. The mean CRP level and leukocytes count were $6.5 \pm 5.5 \mathrm{mg} /$ $\mathrm{dl}$ and $13.6 \pm 11.3 \mathrm{mg} / \mathrm{dl}$ respectively. Median logistic EuroSCORE was $15.3 \%$ (CI 9.3-18.8) and EuroSCORE II 4.84\% (CI 2.9-7.9) (Table 2). The most affected valve was the tricuspid valve $(62.5 \%, n=15)$. Four patients (16.7\%) underwent a multivalve procedure (Table 3). Overall cumulative survival at 30 days, 1 year and 3 years was 88,78 and $72 \%$ respectively (Fig. 1 ). Freedom from the combined events at secondary endpoint was $83 \%$ at 30 days, $68 \%$ at 1 year and $58 \%$ at 3 years (Fig. 2).

To evaluate independent predictors of 30-day mortality, a logistic regression model was constructed. Several univariate indicators were found to predict 30-day mortality. This model showed that logistic EuroSCORE I (odds ratio [OR], 1.078; 95\% confidence interval [CI], 1.017-1.142; $P=0.012$ ), EuroSCORE II (OR, 1.339; $95 \%$ CI, 1.117-1.606, $P=0.002$ ), history of Liver cirrhosis

Table 1 Preoperative data

\begin{tabular}{ll}
\hline Female & $7(29.2 \%)$ \\
Age, years & $38.5 \pm 8,7$ \\
BMI, kg/m ${ }^{2}$ & $24,7 \pm 4.3$ \\
NYHA-Class & $2.7 \pm 0.6$ \\
Chronic kidney injury & $7(29.2 \%)$ \\
Liver cirrhosis & $4(16.7 \%)$ \\
Hepatitis C & $19(79.2 \%)$ \\
HIV & $3(12.5 \%)$ \\
Positive blood cultures & $17(70.8 \%)$ \\
Staphylococcus aureus & $14(58.3 \%)$ \\
Staphylococcus epidermidis & $1(4.2 \%)$ \\
Enterokokkus spiecies & $2(8.3 \%)$ \\
Leukocytes, mg/dl & $13.55 \pm 11.3$ \\
CRP, mg/dl & $6.5 \pm 5.5$ \\
Septic emlolism & $15(62.5 \%)$ \\
EuroSCORE II, \% & $4.84(2.9-7.9)$ \\
logistic EuroSCORE I, \% & $15,3(9.31-18.8)$ \\
\hline BMI Body Mass Index, CRP C-reactive Protein, HIV Human Immunodeficiency \\
Virus, NYHA New York Heart Association
\end{tabular}


Table 2 Intraoperative data

\begin{tabular}{ll}
\hline Bypass time, min & $95.33 \pm 51.1$ \\
Operating time, min & $178.4 \pm 61.4$ \\
Cross-clamp time, min & $49.13 \pm 39.8$ \\
Lateral thoracotomy & $2(8.3 \%)$ \\
Type of valve surgery & \\
Mechanical prosthesis & $1(4.2 \%)$ \\
Biological prosthesis & $16(66.7 \%)$ \\
Valve repair & $7(29.1 \%)$ \\
Site of valve surgery & \\
Aortic & $6(25 \%)$ \\
Mitral & $7(29.1 \%)$ \\
Tricuspid & $15(62.5 \%)$ \\
Aortic+ mitral valve & $3(12.5 \%)$ \\
Aotric+mitral+tricuspidal & $1(4.2 \%)$ \\
Multivalve surgery & $4(16.7 \%)$ \\
Elective & $3(12.5 \%)$ \\
Urgent & $15(62.5 \%)$ \\
Emergent & $6(25 \%)$ \\
\hline
\end{tabular}

(OR, 8.149; 95\% CI, 1.615-41.12, (to $P=0.011$ ), and positive blood cultures with Enterococcus species (OR, 0.997; 95\% CI, 0.181-5.489, $P=0.03$ ), were significantly associated with 30-day mortality.

\section{Discussion}

In patients presenting with active intravenous drug use, IE is a severe condition associated with high mortality and recurrence rates $[7,8]$. Even though, multiple

Table 3 Postoperative outcomes

\begin{tabular}{ll}
\hline Atrial fibrillation & $4(16.7 \%)$ \\
AVB III & $4(16.7 \%)$ \\
Permanent pacemaker & $2(8.3 \%)$ \\
Acute kidney failure on dialysis & $8(33.3 \%)$ \\
Coagulation disorder & $3(12.5 \%)$ \\
Reopening for bleeding & $2(8.3 \%)$ \\
Cerebrovascular accident & 0 \\
Recurrent IE & $5(20.8 \%)$ \\
Re-operation because of IE & $4(16.7 \%)$ \\
Deep sternal wound infection & $1(4.2 \%)$ \\
Septic shock & $3(12.5 \%)$ \\
Multiorgan failure & $4(16.7 \%)$ \\
Follow-up time, years & $4.2 \pm 4.3$ \\
In-hospital mortality & $3(12.5 \%)$ \\
30-day-mortality & $3(12.5 \%)$ \\
\hline
\end{tabular}

AVB Atrioventricular block, IE Infective endocarditis research groups have published numerous articles on IE in IVDUs, an ongoing debate about the prognosis in this challenging pathology still persists [9]. This report describes our single center experience on the surgical treatment of IE in patients with IVDU over a period of 13 years and analyses the predictors of mortality. The aim of this study is to identify the factors which increase the mortality in this particular cohort. The results of our study could lead to improvement of surgical therapy and postoperative care.

In our cohort, overall cumulative mortality at 30 days, 1 year and 3 years was 12, 22 and 28\% respectively (Fig. 1). A high 30 -day mortality of $12 \%$ is rather atypical for the patients with IVDU according to the most trials conducted by other study groups $[10,11]$. On the other hand, $87.5 \%$ of the patients underwent an urgent or emergent procedure, which could explain the unexpected poor short-term survival. After 1 year, the mortality rate was $22 \%$, which is in line with the results published in the literature. The reason for low short-term mortality rates of the patients with active IVDU is the young patients' age, few comorbidities and more common involvement of the right-sided valves [11-13]. In our study, $62.5 \%$ of patients $(n=15)$ suffered from tricuspid valve endocarditis, which correlates with a rate of right-sided valves involvement as described by Kim at al [11].

Unfortunately, due to their recurrent endocarditis, patients with IVDU suffering from IE have much higher readmission rates when compared with endocarditis patients in the general population [14-16]. Patients with IVDU present with higher mortality between postoperative days 90 and 180 [8]. In our study, readmission due to recurrent IE stands at $22.5 \%(n=5)$ of patients. Four of them $16.7 \%$ underwent a further cardiac procedure for IE and one patient (4.2\%) underwent a successful antibiotic treatment. All patients suffering from the recurrent IE in our cohort also started injecting drugs again.

Recent literature reports the relapse rates of intravenous drug use after hospital discharge at a range between 22 and $49 \%[14,17,18]$. In our cohort, some of the patients were participating in the methadone-program, receiving daily opioid substitute from specialized centers, but at the time of admission to the hospital all of them were still active drug users. Unfortunately, additionally to the medical addiction treatment no patient was offered any psychiatric treatment or had a caring social worker at their side. In one institutional study, more than half of the patients discharged from the hospital were lacking any kind of planned addiction treatment [17]. Due to high relapse rates and the consequent high rates of recurrent IE in patients with active IVDU, the concept of medication associated treatment (MAP) should be adopted. MAP involves medical treatment, 


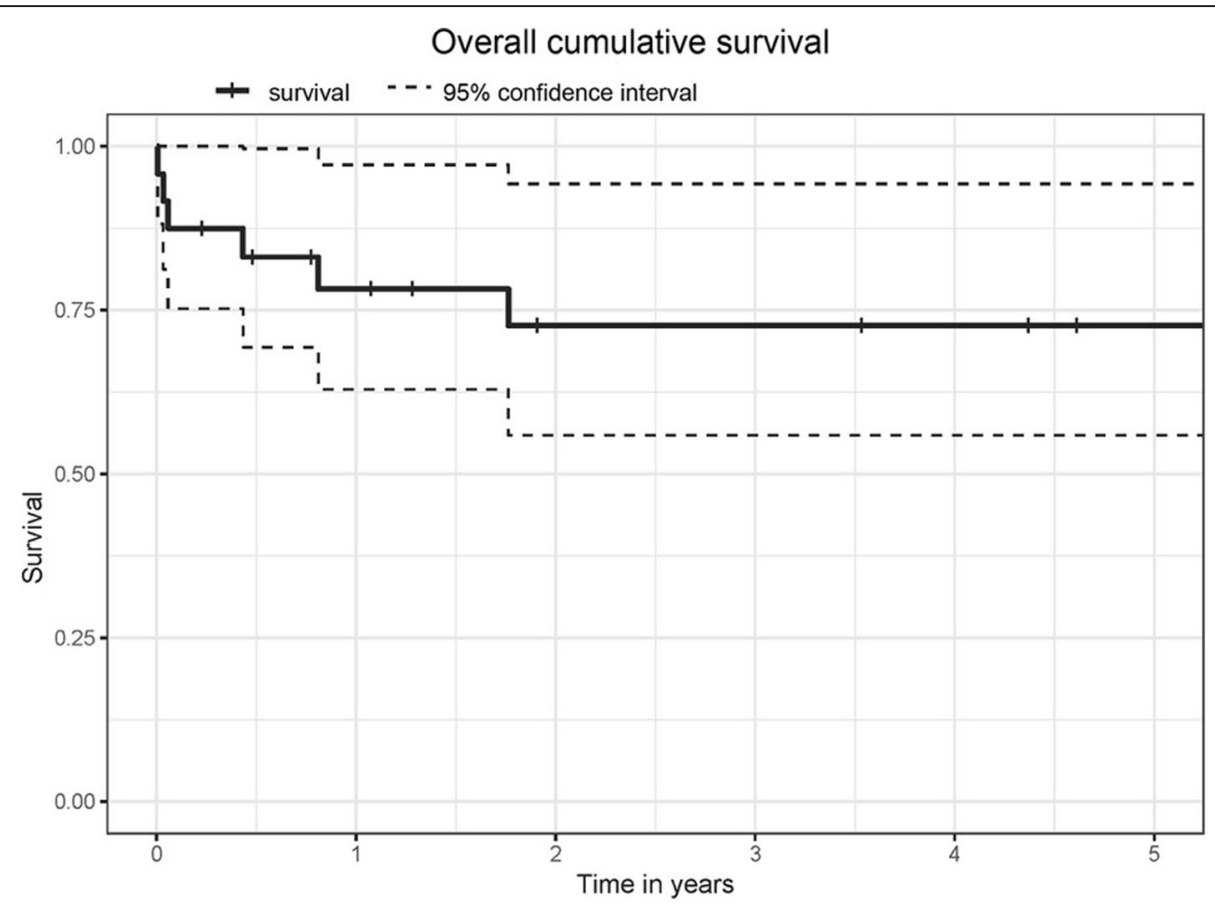

Number at risk

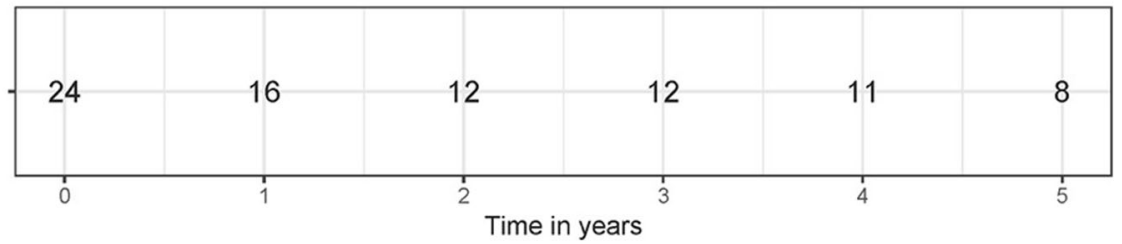

Fig. 1 Cumulative survival

psychiatric evaluation and counseling, which should be considered in every hospital-treated IVDU patient with IE. As reported by Mohlman et al., lower mortality and morbidity rates, lower hospital cost and overall less referrals to different departments of the health system was found in MAP enrolled patients [19]. The current situation is that patients with IVDU are at greater risk of recurrent endocarditis between 3 and 6 months when compared to the patients with no history of drugs abuse, given both population are being treated with antibiotics for 6 weeks postoperatively [20]. The hazard of death after reoperation for recurrent IE in IVDU-patients is 10 times higher than in non-drug-users. Therefore, it is crucial that in this group of patients appropriate addiction therapy is offered along pathogen targeted antibiotic therapy,

Septic shock was at $12.5 \%(n=3)$ the most common cause of death in our cohort. Haidari et al. described the use of the intraoperative hemadsorption in patients with native valve infective endocarditis and illustrated a significant reduction in postoperative sepsis and sepsisassociated deaths in patients who received intraoperative hemadsorption [21]. The use of blood purifying mechanisms such as hemadsorption in patients with acute IE and chronic IVDU could be a way to reduce the number of sepsis-associated postoperative deaths. Moreover, patients receiving hemadsorption seem to show better hemodynamic stability and lower rates of postoperative organ failure [22].

The most common pathogen in our cohort was Staphylococcus aureus (Table 1). Our results match with previously published studies which reported that most patients with IVDU and IE present with Staphylococcus aureus infection [23-25]. Although, some research groups reported increased mortality rates in patients suffering from IE with Staphylococcus aureus infection [26, 27], our cohort has shown that infection with Enterococcus faecalis was the predictive factor for mortality in univariate analysis (Table 4). Antibiotic treatment remains an important part of the endocarditis treatment and in some cases it may be used as the sole treatment modality. In our institution we perform without exceptions a six-week intravenous antibiotic therapy tailored to the pathogen revealed in the bloodstream. There are 


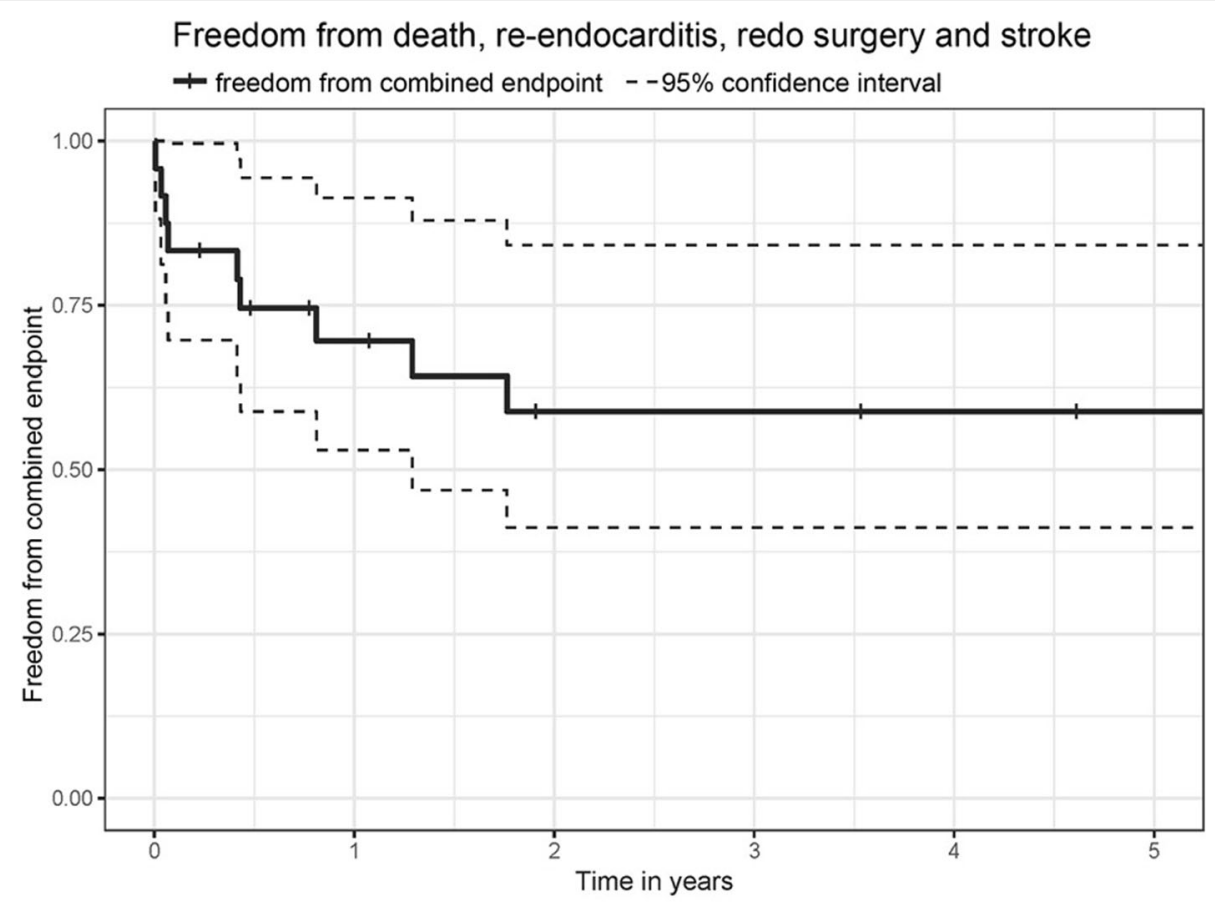

Number at risk

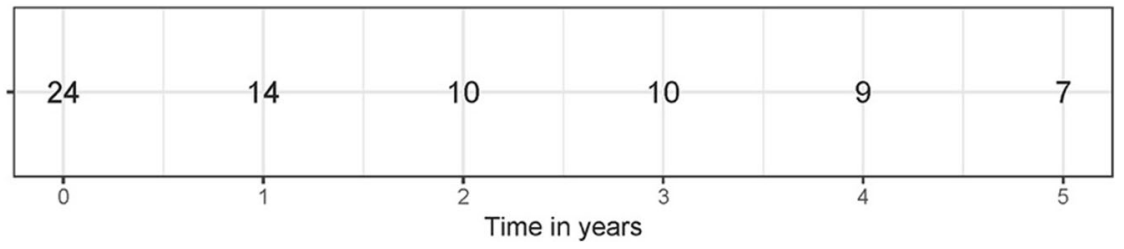

Fig. 2 Freedom from death, re-endocarditis, redo surgery and stroke

reports of abbreviated antibiotic therapy, performed in uncomplicated cases of right sided IE with either oral or intravenous antibiotics. Unfortunately, this concept is usually not suitable for patients undergoing an urgent or emergent procedures with already impaired organ function [28].

There is an ongoing debate about the most precise score to predict the surgical risk in patients with acute IE. In our study, the logistic EuroSCORE and EuroSCORE II appeared to be the mortality predictors in the univariate analysis. However, some research groups are working on alternative scores to predict the operative mortality [29]. Furthermore, liver cirrhosis was an independent mortality predictor in the univariate analysis. This finding can be explained due to a small number of patients and should be definitely reevaluated on a larger population.

In our institution we aim for the radical approach while operating on patients with IE. In some cases, radical surgical debridement may cause severe damage of the conduction system and lead to the complete AV block postoperatively. In our cohort there were 4 patients presenting with AV-Bloc III postoperatively with two of them (8.3\%) requiring a permanent pacemaker. Because of the relatively low number of patients, further studies on larger cohorts need to be performed to support these findings.

There is a constant contradiction on the type of the valve prosthesis to use in patients with acute IE and especially in patients with IVDU, who often lack compliance.

At our institution, we tend to repair the right-sided valves and replace the left-sided valves. The current guidelines on valve surgery recommend to go for a valve repair, if possible, also in cases of IE [30]. There are various techniques of tricuspid valve repair in patients with acute IE [31, 32]. There are contradicting evidences on the type of the valve prosthesis used in case of potential valve replacement. The current state is that there is no difference in survival or recurrence of IE after using biological or mechanical valve prosthesis [33]. In our cohort, despite of the young patients' age, we implanted biological prostheses in $66.7 \%$ of cases to avoid anticoagulation regimen, given a questionable compliance of the patients with active IVDU. Regardless of the surgical 
Table 4 Independent risk factors of 30-day mortality - univariate logistic regression

\begin{tabular}{|c|c|c|}
\hline Characteristics & Odds ratio with $95 \%$ Confidence interval & $p$-value \\
\hline Female gender & 0.384 (0.045 to 3.306$)$ & 0.383 \\
\hline Age & 1.022 (0.926 to 1.127$)$ & 0.672 \\
\hline Logistic EuroSCORE I & $1.078(1.017$ to 1.142$)$ & 0.012 \\
\hline EuroSCORE II & 1.339 (1.117 to 1.606$)$ & 0.002 \\
\hline NYHA Class & 1.445 (0.378 to 5.527$)$ & 0.591 \\
\hline Kidney disease & 2.844 (0.571 to 14.166$)$ & 0.202 \\
\hline GFR & 0.973 (0.931 to 1.017$)$ & 0.225 \\
\hline Creatinine & 1.151 (0.299 to 4.432$)$ & 0.838 \\
\hline Liver cirrhosis & 8.149 (1.615 to 41.118$)$ & 0.011 \\
\hline Bilirubin & 1.890 (0.782 to 4.563$)$ & 0.157 \\
\hline Hepatitis C & 1.456 (0.170 to 12.472$)$ & 0.732 \\
\hline HIV & $0.040(0.01$ to 1560.464$)$ & 0.552 \\
\hline Large vegetation & $2.160(0.252$ to 18.527$)$ & 0.483 \\
\hline Septic embolism & 1.307 (0.239 to 7.145$)$ & 0.758 \\
\hline Positive blood culture & 0.997 (0.181 to 5.489$)$ & 0.997 \\
\hline Negative blood culture & 0.398 (0.046 to 3.433$)$ & 0.402 \\
\hline Staphylococcus species & 0.337 (0.068 to 1.839$)$ & 0.209 \\
\hline Enterococcus species & 7.348 (1.218 to 44.323$)$ & 0.030 \\
\hline Operation time & 0.998 (0.984 to 1.012 ) & 0.780 \\
\hline Cross clamp time & 1.002 (0.983 to 1.021$)$ & 0.829 \\
\hline Multivalvular endocarditis & 0.046 (0.0 to416017.507) & 0.706 \\
\hline
\end{tabular}

HIV Human immunodeficiency virus, GFR Glomerular filtration rate, NYHA New York Heart Association Functional Classification

method, many surgeons are less likely to operate on patients with IE and IVDU due to an increased risk of IE recurrence and its associated higher operative mortality and a lower success of antibiotic treatment [11].

\section{Conclusion}

In patients presenting with active intravenous drug abuse, treatment of infective endocarditis should be performed as aggressively as possible followed by antibiotic therapy to avoid high mortality rates due to recurrent endocarditis. Early intervention is advisable in patients with an infective endocarditis and enterococcus species in the preoperative blood cultures, liver function deterioration as well as cardiac function impairment, as these factors predict 30-day mortality. Attention should be also payed to addiction treatment, due to the elevated relapse rate in patients who actively inject drugs. However, larger prospective studies are necessary to support our results. As septic shock is the most frequent cause of death, new treatment options, e.g. blood purification should be evaluated.

\section{Study limitations}

We have to report on several study limitations. Alongside with the observational non-randomized type of study from a single center, we were limited by the number of patients. Because of the rareness of the described condition in the clinical practice, we were not able to provide a larger size of the sample, so further multicenter clinical studies have to be performed to allow for generalizability of the results. Moreover, this kind of clinical studies is strongly limited by its' ethical unfeasibility, as a direct comparison of the surgical and medical treatment is required to analyze the results and increase the statistical power. Another important point we have to address is that the patients admitted were referred from different hospitals, therefore making it impossible to determine the onset time of the IE in each case.

\section{Acknowledgements}

The authors thank the patient for the consent to this publication.

Disclosures

None.

Authors' contributions

$A Z, M S, A O, J E, A A R$ und RV contributed to this manuscript: case management, surgical procedure, literature research and writing the manuscript. KZ, AR, BS and AW, contributed to this manuscript: literature research, case management and critical revision of the manuscript. All authors read and approved the final manuscript. 


\section{Funding}

The authors declare the received no funding for the research reported. Open Access funding enabled and organized by Projekt DEAL.

\section{Availability of data and materials}

The datasets used and analysed during the current study are available from the corresponding author on reasonable requests.

\section{Declarations}

\section{Ethics approval and consent to participate}

The study was approved by the local ethics committee (reference number 20-9289-BO). The authors thank the patients for the consent to publication of the data.

\section{Consent for publication}

The authors thank the patients for informed consent to publication of the data.

\section{Competing interests}

None declared.

\section{Author details}

'Department of Thoracic and Cardiovascular Surgery, West German Heart and Vascular Center, University of Duisburg-Essen, Essen, Germany. ${ }^{2}$ Department of Medicine, Faculty of Medicine, Imperial College London, London, UK

Received: 23 February 2021 Accepted: 6 April 2021

Published online: 20 April 2021

\section{References}

1. Degenhardt L, Peacock A, Colledge S, Leung J, Grebely J, Vickerman P, et al. Global prevalence of injecting drug use and sociodemographic characteristics and prevalence of HIV, HBV, and HCV in people who inject drugs: a multistage systematic review. Lancet Glob Heal. 2017;5(12):e1192207. https://doi.org/10.1016/S2214-109X(17)30375-3.

2. Chu VH, Park LP, Athan E, Delahaye F, Freiberger $T$, Lamas $C$, et al. Association between surgical indications, operative risk, and clinical outcome in infective endocarditis a prospective study from the international collaboration on endocarditis. Circulation. 2015;131(2):13140.

3. Moreillon P, Que YA. Infective endocarditis. In: Lancet; 2004

4. Li JS, Sexton DJ, Mick N, Nettles R, Fowler VG, Ryan T, et al. Proposed Modifications to the Duke Criteria for the Diagnosis of Infective Endocarditis. Clin Infect Dis. 2000;30(4):633-8.

5. Singer M, Deutschman CS, Seymour CW, Shankar-Hari M, Annane D, Bauer $M$, et al. The third international consensus definitions for Sepsis and septic shock (Sepsis-3). JAMA. 2016;315(8):801-10. https://doi.org/10.1001/jama.201 6.0287 .

6. Hilton R. Defining acute renal failure. C Can Med Assoc J. 2011;183(10):1167-9.

7. Habib G. Management of infective endocarditis. Heart. 2006;92(1):124-30. https://doi.org/10.1136/hrt.2005.063719.

8. Shrestha NK, Jue J, Hussain ST, Jerry JM, Pettersson GB, Menon V, et al. Injection drug use and outcomes after surgical intervention for infective endocarditis. Ann Thorac Surg. 2015;100(3):875-82.

9. Ortiz-Bautista C, López J, García-Granja PE, Sevilla T, Vilacosta I, Sarriá C, et al. Current profile of infective endocarditis in intravenous drug users: prognostic relevance of the valves involved. Int J Cardiol. 2015;187:472-4. https://doi.org/10.1016/j.ijcard.2015.03.368.

10. Ribera E, Miró JM, Cortés E, Cruceta A, Merce J, Marco F, et al. Influence of human immunodeficiency virus 1 infection and degree of immunosuression in the clinical characteristics and outcome of infective endocarditis in intravenous drug users. Arch Intern Med. 1998;158(18):2043-50. https://doi. org/10.1001/archinte.158.18.2043.

11. Kim JB, Ejiofor Jl, Yammine M, Ando M, Camuso JM, Youngster I, et al. Surgical outcomes of infective endocarditis among intravenous drug users. In: Journal of Thoracic and Cardiovascular Surgery; 2016.

12. Zubarevich A, Szczechowicz M, Brcic A, Osswald A, Tsagakis K, Wendt D, et al. Tricuspid valve repair in isolated tricuspid pathology: a 12-year single center experience. J Cardiothorac Surg. 2020;15(1):330. https://doi.org/10.11 86/s13019-020-01369-8.

13. Farag M, Borst $T$, Sabashnikov A, Zeriouh M, Schmack B, Arif R, et al. Surgery for infective endocarditis: outcomes and predictors of mortality in 360 consecutive patients. Med Sci Monit. 2017;23:3617-26. https://doi.org/10.12 659/MSM.902340.

14. Rudasill SE, Sanaiha Y, Mardock AL, Khoury H, Xing H, Antonios JW, et al. Clinical Outcomes of Infective Endocarditis in Injection Drug Users. J Am Coll Cardiol. 2019;73(5):559-70

15. Szczechowicz M, Weymann A, Mkalaluh S, Mashhour A, Zhigalov K, Easo J. Surgical options for aortic root replacement in destructive endocarditis. Brazilian J Cardiovasc Surg. 2020;35(3):265-73. https://doi.org/10.21470/1 678-9741-2020-0020

16. Easo J, Szczechowicz M, Hölzl P, Meyer A, Zhigalov K, Malik R, et al. Stentless root replacement versus tissue valves in infective endocarditis - a propensity-score matched study. Brazilian J Cardiovasc Surg. 2020;35(4):4119. https://doi.org/10.21470/1678-9741-2020-0267.

17. Gray ME, Rogawski McQuade ET, Scheld WM, Dillingham RA. Rising rates of injection drug use associated infective endocarditis in Virginia with missed opportunities for addiction treatment referral: a retrospective cohort study. BMC Infect Dis. 2018;18(1):532. https://doi. org/10.1186/s12879-018-3408-y.

18. Rosenthal ES, Karchmer AW, Theisen-Toupal J, Castillo RA, Rowley CF. Suboptimal Addiction Interventions for Patients Hospitalized with Injection Drug Use-Associated Infective Endocarditis. Am J Med. 2016; 129(5):481-5.

19. Mohlman MK, Tanzman B, Finison K, Pinette M, Jones C. Impact of medication-assisted treatment for opioid addiction on Medicaid expenditures and health services utilization rates in Vermont. J Subst Abus Treat. 2016;67:9-14. https://doi.org/10.1016/j.jsat.2016.05.002.

20. Baddour LM. Twelve-year review of recurrent native-valve infective endocarditis: a disease of the modern antibiotic era. Clin Infect Dis. 1988; 10(6):1163-70. https://doi.org/10.1093/clinids/10.6.1163.

21. Haidari Z, Wendt D, Thielmann M, Mackowiak M, Neuhäuser M, Jakob H, et al. Intraoperative Hemoadsorption in patients with native mitral valve infective endocarditis. In: Annals of Thoracic Surgery; 2020.

22. Träger K, Skrabal C, Fischer G, Datzmann T, Schroeder J, Fritzler D, et al. Hemoadsorption treatment of patients with acute infective endocarditis during surgery with cardiopulmonary bypass - a case series. Int J Artif Organs. 2017;40(5):240-9. https://doi.org/10.5301/ijao.5000583.

23. DiNubile MJ. Abbreviated therapy for right-sided Staphylococcus aureus endocarditis in injecting drug users: the time has come? European journal of Clinical Microbiology \& Infectious Diseases; 1994.

24. Hecht SR, Berger M. Right-sided endocarditis in intravenous drug users. Prognostic features in 102 episodes. Ann Intern Med. 1992;117(7):560-6.

25. Mathew J, Addai T, Anand A, Morrobel A, Maheshwari P, Freels S. Clinical Features, Site of Involvement, Bacteriologic Findings, and Outcome of Infective Endocarditis in Intravenous Drug Users. Arch Intern Med. 1995; 155(15):1641-8.

26. Fowler VG, Miro JM, Hoen B, Cabell CH, Abrutyn E, Rubinstein E, et al. Staphylococcus aureus endocarditis: a consequence of medical progress. J Am Med Assoc. 2005;293(24):3012-21. https://doi.org/10.1001/jama.293.24.3 012.

27. Cabell CH, Jollis JG, Peterson GE, Ralph Corey G, Anderson DJ, Sexton DJ, et al. Changing patient characteristics and the effect on mortality in endocarditis. Arch Intern Med. 2002;162(1):90-4.

28. Baddour LM, Wilson WR, Bayer AS, Fowler VG, Tleyjeh IM, Rybak MJ, et al. Infective endocarditis in adults: diagnosis, antimicrobial therapy, and management of complications: a scientific statement for healthcare professionals from the American Heart Association. Circulation. 2015;132(15): 1435-86. https://doi.org/10.1161/CIR.0000000000000296.

29. Urso S, Tena MÁ, Horcajada I, Paredes F, González-Barbeito M, Portela F. Prediction of surgical risk in patients with endocarditis: comparison of logistic EuroSCORE, EuroSCORE II and APORTEI score. Enferm Infecc Microbiol Clin. 2020;50213-005X(20):30209-3.

30. Baumgartner H, Falk V, Bax JJ, De Bonis M, Hamm C, Holm PJ, et al. 2017 ESC/EACTS guidelines for the Management of Valvular Heart Disease. Rev Esp Cardiol (Engl Ed). 2018:71(2):110.

31. Dawood MY, Cheema FH, Ghoreishi M, Foster NW, Villanueva RM, Salenger $R$, et al. Contemporary outcomes of operations for tricuspid valve infective endocarditis. Ann Thorac Surg. 2015;99(2):539-46. 
32. Gottardi R, Bialy J, Devyatko E, Tschernich H, Czerny M, Wolner E, et al. Midterm Follow-Up of Tricuspid Valve Reconstruction Due to Active Infective Endocarditis. Ann Thorac Surg. 2007;84(6):1943-8.

33. Toyoda N, Itagaki S, Tannous H, Egorova NN, Chikwe J. Bioprosthetic versus mechanical valve replacement for infective endocarditis: focus on recurrence rates. Ann Thorac Surg. 2018;106(1):99-106.

\section{Publisher's Note}

Springer Nature remains neutral with regard to jurisdictional claims in published maps and institutional affiliations.

Ready to submit your research? Choose BMC and benefit from:

- fast, convenient online submission

- thorough peer review by experienced researchers in your field

- rapid publication on acceptance

- support for research data, including large and complex data types

- gold Open Access which fosters wider collaboration and increased citations

- maximum visibility for your research: over $100 \mathrm{M}$ website views per year

At $\mathrm{BMC}$, research is always in progress.

Learn more biomedcentral.com/submissions 